\title{
Tramando futuros. Transnacionalismo familiar en la migración desde República Dominicana y Brasil hacia España
}

\author{
Claudia PEDONE \\ CONICET- Instituto Interdisciplinario de Estudios de Género, \\ Facultad de Filosofía y Letras, Universidad de Buenos Aires \\ claudiapedone@yahoo.es \\ Sandra GIL ARAUJO \\ CONICET- Instituto de Investigaciones Gino Germani, \\ Facultad de Ciencias Sociales, Universidad de Buenos Aires \\ sandragilaraujo@yahoo.es
}

Recibido: Septiembre 2015

Aceptado: Marzo 2016

\section{RESUMEN}

En este artículo exploramos las formas de re-organización familiar desplegadas por los y las migrantes en el marco de una (nueva) crisis económica global y de endurecimiento del control migratorio en Europa, tomando como casos de estudio las migraciones de Brasil y República Dominicana hacia España.

Nuestra finalidad no es caracterizar la totalidad de estrategias de estos colectivos, sino visibilizar su heterogeneidad. Los desplazamientos de población dominicana y brasileña hacia España tienen en común el rol de las mujeres como primer eslabón de las cadenas migratorias. En ambos casos las mujeres son el sostén económico de las familias transnacionales y lideran los procesos de reagrupación. Sin embargo, las diferencias en el tiempo transcurrido en el país de destino, el estatus jurídico, la procedencia (urbana-rural), el nivel formativo, la pertenencia de clase y la inserción laboral en destino, inciden de modo diferencial en el diseño y puesta en marcha de los proyectos migratorios, en la organización del cuidado y en las estrategias de reunificación familiar. Estos hallazgos cuestionan el lugar preponderante que se le suele otorgar al origen nacional en el estudio de los procesos migratorios internacionales.

Palabras claves: Migración brasileña, migración dominicana, España, transnacionalismo familiar. 


\title{
Weaving Futures. Family Transnationalism in the migration from Dominican Republic and Brazil to Spain
}

\begin{abstract}
This article explores forms of migrant families' reorganization within a (new) global economic crisis and the hardening of migration control in Europe; based on the cases of Dominican and Brazilian migration to Spain.

Our goal is not to characterize the wholeness of strategies from these collectives, instead visualize its heterogeneity. Displacement of Dominican and Brazilian population to Spain shares the role of women as the first link of migration chains. In both cases women are the economic support of transnational families and they lead reunification's processes. Nevertheless, differences in the time spent in the destination country, migratory status, origin (rural-urban), level of education, class and labor insertion in destination country, affect differently, the planning and start up of migration projects, the organization of care and family reunification strategies. These findings question the predominant place granted to national origin in the study of international migration.
\end{abstract}

Keywords:Brazilian migration, Dominican migration, Spain, Family Transnationalism.

\section{INTRODUCCIÓN}

A mitad de los años ochenta España se configuró como destino alternativo para las migraciones latinoamericanas, en el marco del endurecimiento del control migratorio en Estados Unidos y las crisis que atravesaron las economías del Continente, en contraste con las transformaciones experimentadas por España en el seno de la Comunidad Europea. En el inicio del siglo XXI América Latina se convirtió en principal lugar de origen de la migración asentada en España. La aceleración y feminización fueron dos de los rasgos distintivos de estas corrientes, en especial las provenientes de Ecuador, Colombia, República Dominicana y Brasil, posteriormente de Bolivia y Paraguay, y en los últimos años Honduras.

La migración masiva de República Dominicana hacia Estados Unidos, principalmente a Nueva York, comenzó en 1962, después de la muerte del Dictador Rafael Trujillo que puso fin a una política que obstaculizaba la emigración. Si bien en una primera etapa tenía un carácter político, con el tiempo se convirtió en una migración con fuerte trasfondo económico. A mediados de 1980, en el marco del deterioro de las condiciones económicas en República Dominicana, España se perfiló en un destino de migración. Mientras se hacía cada vez más difícil migrar a Estados Unidos por vías regulares, en España se podía ingresar sin visado. Esto se modificó en el año 1992. Otros factores que impulsaron estos movimientos de población fueron las relaciones económicas y culturales entre España y República Dominicana, que incrementaron los flujos de bienes, servicios y capitales entre ambos países, y por supuesto, los desplazamientos y los lazos laborales y familiares (Herranz, 1997; Gregorio Gil, 1998; Ariza, 2000). En cuanto al perfil de la población dominicana en España, las migrantes pioneras fueron estudiantes, pero a fines de la década de 1980 el $85 \%$ eran mujeres de origen rural que migraban solas encabezando proyectos 
familiares y se insertaban principalmente en el trabajo doméstico (Gallardo 1995). Esta migración aumentó notoriamente en la década siguiente. En enero de 2002, 45.887 personas nacidas en República Dominicana estaban empadronadas en España, con un $69 \%$ de componente femenino (INE) ${ }^{1}$. En la actualidad continúa siendo una población muy joven, el $70 \%$ tiene entre 15 y 40 años. Se concentran en los núcleos urbanos, principalmente Madrid y en segundo lugar Barcelona. Su nivel educativo es superior a la media de República Dominicana, pero inferior al de la población española y al resto de migrantes de América Latina que residen en España. En cuanto a la situación jurídica, los porcentajes de irregularidad de la migración dominicana son bajos debido a la imposición del visado, los diversos procesos de regularización y la alta tasa de nacionalizaciones, que han agilizado las reagrupaciones familiares (García O’Meany, 2009). Si bien con el tiempo la reagrupación familiar ha generado un aumento de la presencia de varones, esta migración aún tiene un protagonismo femenino. En enero de 2009 residían en España 86.446 habitantes con nacionalidad dominicana (57\% mujeres). Pero al prestar atención a los empadronados nacidos en República Dominicana la cifra llegaba a 129.751 (61\% mujeres) y ascendía a 136.803 en el año 2010. El 35\% tenía nacionalidad española, de los cuales el 67\% eran mujeres. En enero de 2015 el número de empadronados con nacionalidad dominicana descendió a 75.315 (57\% mujeres), al tiempo que la cifra de los nacidos en la República Dominicana siguió aumentando a 161.232 (61\% mujeres). De este total, el 54\% tiene nacionalidad española, $61 \%$ de los cuales son mujeres. Además de los altos porcentajes de nacionalizados, estos datos indican que el número de empadronados nacidos en República Dominicana siguió creciendo en los años de crisis.

Estados Unidos también ha sido el destino primordial de la migración brasileña, especialmente los estados de Nueva York, Massachussets, Florida, Connecticut y California (Sales, 2004; Ribeiro 1998; Margolis, 1994). Si bien en los inicios fue una migración encabezada por varones, a partir de procesos de reagrupación familiar y de la consolidación de las redes migratorias se observa una mayor presencia de familias. El aumento del flujo migratorio brasileño hacia Europa, en general, y hacia España, en particular, coincidió con el reposicionamiento económico y político de Brasil en el mercado internacional como "país emergente". Brasil, al igual que otros países del Cono Sur de América Latina, se ha caracterizado históricamente como un país de inmigración, fenómeno que ha desempeñado un importante papel en la formación y composición de la sociedad brasileña. A partir de la década de 1980, el patrón migratorio de Brasil cambia rotundamente y aunque continuó recibiendo migrantes de América Latina, su población comenzó a emigrar hacia Estados Unidos, Portugal, Japón y, posteriormente, a España, Italia y Reino Unido.

En el caso de España, la migración de Brasil se incrementó de modo notable durante la primera década del siglo XXI. De 19.828 personas de nacionalidad brasileña

1 Todos los datos de empadronamiento han sido obtenidos a principios de marzo de 2016, mediante el cruce de variables de la base de datos del padrón de habitantes del Instituto Nacional de Estadística, disponibles en su página web.http://www.ine.es 
empadronadas al 1 de enero de 2002, se pasó a 110.124 en enero del 2009². Entre los rasgos distintivos de este flujo está la feminización, una procedencia regional variada y una composición de clase heterogénea (Carvalho, 2010). Del 2002 al 2009 el porcentaje de feminización decreció del 69\% al 61\%.El aumento de la proporción de varones se relaciona con la puesta en marcha de procesos de reunificación familiar liderados por mujeres, tanto por la vía formal como autónoma, ya que los migrantes brasileños no necesitan visado para ingresar como turistas al espacio Schengen. La exención de visado se vincula también con los altos índices de irregularidad de este colectivo, que para el año 2006, después del último proceso de regularización, superaba el 60\%. En enero de 2010, año en que comenzó nuestro estudio, estaban empadronadas en España 103.049 personas con nacionalidad brasileña, de las que 63\% eran mujeres. La cifra llegaba a 132.651 si atendemos a los nacidos en Brasil. En cuanto a la distribución geográfica, la mayor parte se asienta en Cataluña (22\%), Madrid (20\%), y, en menor medida, en Galicia y Andalucía (10\%). Pero en términos relativos la población brasileña tiene más peso en Galicia, ya que representa el 10\% del total de población extranjera empadronada en esa comunidad autónoma, en comparación con el 2\% en Madrid y Barcelona, según los datos del padrón municipal de 2010. Si prestamos atención al lugar de nacimiento su peso es aún más significativo, ya que un tercio de la población de origen brasileño que reside en Galicia tiene nacionalidad española. Esto explica porque parte de nuestro trabajo de campo se realizó en Galicia. Según los últimos datos del padrón de habitantes, en julio de 2015 residían en España (de forma regular e irregular) 57.347 personas con nacionalidad brasilera (67\% mujeres). Al prestar atención a Brasil como país de nacimiento, la cifra llega a 100.202 (65\% mujeres). Estos datos dan cuenta de la importante cantidad de personas de origen brasileño que poseen una nacionalidad distinta a la de nacimiento. También confirman el descenso de migrantes brasileños empadronados en España y el aumento de la proporción de mujeres en este flujo a partir del 2009, en el contexto de agudización de la crisis económica mundial.

Estos breves perfiles verifican que los desplazamientos de población dominicana y brasileña hacia España tienen en común el rol de las mujeres como primer eslabón de las cadenas migratorias. En ambos casos las mujeres son el sostén económico de las familias transnacionales y lideran los procesos de reagrupación. Sin embargo, las diferencias en el tiempo transcurrido en el país de destino, el estatus jurídico, la procedencia (urbana-rural), el nivel formativo, la pertenencia de clase y la inserción laboral en destino, inciden de modo diferencial en el diseño y puesta en marcha de los proyectos migratorios, en la organización del cuidado y en las estrategias de reunificación familiar.

Considerando estos antecedentes, en este artículo nos proponemos visibilizar la heterogeneidad que caracteriza las formas de organización familiar en el contexto de la migración entre América Latina y Europa, a partir del estudio de la migración de

\footnotetext{
${ }^{2}$ Todos los datos de empadronamiento han sido obtenidos mediante el cruce de variables de la base de datos del padrón de habitantes del Instituto Nacional de Estadística, disponibles en su página web. http://www.ine.es
} 
República Dominicana y Brasil hacia España. Cuando en nuestras investigaciones nos referimos a los cambios en las formas de organización familiar en el contexto de la migración queremos resaltar fundamentalmente dos aspectos, vinculados entre sí. Por un lado, la dispersión geográfica de los integrantes de la familia que supone la migración, y que obliga a "producir" familia a la distancia. Si los integrantes de la familia no viven bajo el mismo techo, ni siquiera en el seno de la misma nación: ¿Cómo se sostienen y recrean esos vínculos familiares? ¿Quiénes los sostienen? ¿Cómo se modifican a lo largo del tiempo? ¿Qué papel tiene la política migratoria en la prolongación o perpetuación de ese distanciamiento, y en las estrategias/formas de residencia de los distintos integrantes de la familia? Por otro lado, atendemos a la incidencia de la migración en la división del trabajo productivo y reproductivo al interior de las familias, en relación al género y la generación de sus integrantes, y nos interesa mostrar cómo esa división cambia a lo largo del tiempo, es decir, a lo largo del curso de vida, del mismo modo que cambia el proyecto migratorio. Y aquí queremos insistir en la importancia de incluir no sólo la dimensión espacial, sino también la dimensión temporal en los análisis sobre las formas de organización familiar en contextos migratorios, ya que el ciclo de vida incide tanto en las configuraciones familiares como en los patrones migratorios. Por este motivo, tanto en las investigaciones individuales como en las desarrolladas en el marco del Grupo Interdisciplinario de Investigadoras Migrantes (GIIM) sobre las migraciones de Ecuador (Pedone, 2006, 2014) y Colombia (Echeverri, 2010; 2014) hacia España, hemos enfatizado la dimensión temporal para abordar las transformaciones de las relaciones familiares en un contexto transnacional y los cambios en los roles y en las relaciones afectivas entre sus miembros. Esta perspectiva la hemos afianzado metodológicamente con un trabajo de campo etnográfico en origen y destino y de corte longitudinal, al trabajar con las mismas familias por más de una década de migración.

Esta investigación se enmarca en el proyecto "Políticas migratorias, transnacionalismo familiar y estratificación cívica. Las migraciones latinoamericanas hacia España”3. El estudio es cualitativo, con un trabajo de campo transnacional, en origen y destino. Hemos entrevistado a i) funcionarios, especialistas, miembros de ONG y asociaciones de inmigrantes en España; ii) en República Dominicana (Santo Domingo) y Brasil (Río de Janeiro), entrevistamos a familiares de migrantes residentes en Madrid, Barcelona y La Coruña (mujeres que se hacen cargo de sus nietos/sobrinos y madres con sus hijos/as adultos/as residentes en España); y iii) entrevistas en profundidad realizadas a migrantes dominicanas/os residentes en Barcelona, en una investigación longitudinal en el marco de distintos proyectos y iv) entrevistas a migrantes brasileñas en La Coruña y Barcelona, en un estudio de carácter exploratorio ${ }^{4}$.

${ }^{3}$ Financiado por el Ministerio español de Ciencia e Innovación, MICIIN, I+D+I (CSO20091349 (subprograma SOCI) (2009-2012), CIIMU, IP. Claudia Pedone.

${ }^{4}$ Las entrevistas a migrantes de Brasil se realizaron entre el 2010 y 2012. Se tuvieron en cuenta los proyectos migratorios y las regiones de origen. En destino realizamos 1 entrevista familiar padre e hija de Matogroso; 2 entrevistas a parejas (Río de Janeiro y de Mina Gerais); 4 
A partir de la revisión de investigaciones sobre transnacionalismo familiar entre España y América Latina consignada en textos precedentes ${ }^{5}$, a continuación resumimos los procesos, dinámicas y actores que por el momento no han sido objeto de una indagación sistemática. También reseñamos los principales estudios sobre la migración de República Dominicana y Brasil a Estados Unidos y al Sur de Europa. En el segundo apartado presentamos nuestro análisis sobre las formas de organización familiar en el contexto de la migración de República Dominicana y Brasil hacia España. Con tal fin prestamos atención a tres de los aspectos que inciden en la re-organización de las familias en los contextos de migración: los proyectos migratorios, la organización del cuidado y las estrategias de reagrupación. Cerramos el artículo con unas breves conclusiones en las que destacamos los hallazgos más originales de nuestra investigación.

\section{MIGRACIÓN, FAMILIA Y TRANSNACIONALISMO: NUEVAS FORMAS DE ORGANIZACIÓN FAMILIAR Y CUIDADOS EN DEBATE}

La aplicación de la perspectiva de género al estudio de las migraciones y el incremento de la presencia de mujeres como primer eslabón de las cadenas migratorias convirtieron a las familias migrantes en objeto de atención de las ciencias sociales, en especial en lo que se refiere a la organización del cuidado de los/as hijos/as que quedaban en el país de emigración. En este marco se ha forjado el concepto de "cuidado transnacional", entendido como el intercambio de cuidado y apoyo que trasciende la distancia geográfica y las fronteras del estado nación (Baldassar, Baldock y Wilding, 2007).

En Europa, la investigación sobre los efectos que tiene la migración internacional en las configuraciones familiares comenzó en la década de los 2000. La familia transnacional ha sido definida como aquella en la cual sus miembros viven la mayor parte del tiempo dispersos entre dos o más países, pero aun así se mantienen unidos por lazos emocionales y/o financieros (Bryceson y Vuorela, 2002). En España, los estudios de los años noventa se enfocaron en las trayectorias socio-laborales de las mujeres

entrevistas con mujeres (2 de Rio de Janeiro, 1 de San Pablo y 1 de Curitiba). En origen hicimos 2 Entrevistas familiares (padre/madre-hija) y 3 entrevistas individuales con familiares de migrantes. Entrevistamos también a informantes claves: 2 en origen y 3 en destino. Las entrevistas con migrantes de República Dominicana fueron realizadas entre el año 2007 y 2011 teniendo en cuenta momento de llegada, estatus administrativo y vínculos familiares. En Barcelona entrevistamos: 2 madres y 2 hijas adolescentes en 2007; 3 mujeres y 2 varones en 2010, 7 mujeres y 2 varones en 2011. Ese mismo año entrevistamos en origen a 3 abuelas, 1 esposa y madre y 1 hijo de migrantes y 4 informantes clave.

${ }^{5}$ Para un estado de la cuestión sobre el abordaje teórico-metodológico del transnacionalismo familiar latinoamericano en el debate académico español consultar Pedone, 2011. Para un estado del arte sobre los principales antecedentes en el estudio del transnacionalismo familiar en el contexto migratorio, con especial atención a las investigaciones que han analizado las dinámicas familiares en la migración entre América Latina y Europa del Sur, ver Gil Araujo y Pedone, 2014. 
migrantes y pasaron en la década siguiente a centrarse en los vínculos familiares entre origen y destino, es decir, en las familias transnacionales (Oso y Ribas, 2012). Desde entonces, las investigaciones sobre la migración de América Latina al Sur de Europa en perspectiva transnacional y de género componen un importante corpus de conocimiento acerca de los vínculos familiares que se generan en estos campos sociales transnacionales. La mayor parte de estos estudios han analizado la migración procedente de Ecuador, Colombia, y, más tarde, Bolivia hacia España (Gil Araujo y Pedone 2014). Son escasos los trabajos sobre vínculos familiares transnacionales en la migración de República Dominicana (Sorensen y Guarnizo 2007; Pedone, Agrela y Gil Araujo 2012) y, principalmente, de Brasil hacia España.

En los últimos años se ha comenzado a indagar las formas que adquiere la paternidad a distancia (Pedone, 2008) y el papel de las abuelas en la organización del cuidado en los lugares de origen y/o destino (Bastia, 2009; Micolta León y Escobar Serrano, 2010). Aún son incipientes los estudios sobre las vinculaciones familiares mantenidas por madres y padres de migrantes adultos jóvenes, o de los niños y adolescentes con sus abuelas, tías y otros integrantes de la familia extensa (Pedone, 2014; Herrera, 2013). También son recientes las investigaciones que analizan los vínculos transnacionales y el impacto de la migración en las formas de organización del cuidado desde la óptica de quienes permanecen en los lugares de emigración (Puyana, Motoa y Viviel 2009; Rivas y Gonzálvez 2009; Gonzálvez 2010; Herrera 2013; Ledo García; 2014). Otro campo muy poco explorado es el de las imbricaciones entre las prácticas y discursos del Estado y la organización transnacional de las familias migrantes entre América Latina y España. Entre los pocos antecedentes en esta línea están los artículos de Pedone y Gil Araujo (2008) y Herrera (2008). Nuestras indagaciones muestran que tanto los proyectos migratorios como las formas de organización de las familias migrantes están fuertemente condicionadas por las políticas migratorias, en concreto las exigencias o no de visado, las regulaciones de la reagrupación familiar y el acceso a la nacionalización (Gil Araujo 2009; Pedone, Agrela Romero y Gil Araujo, 2012; Pedone, Echeverri y Gil Araujo, 2014). En el contexto de la crisis económica que afecta de manera especial a España, se ha comenzado a estudiar la reconfiguración de los proyectos migratorios y la consecuente reorganización de las familias migrantes. Estos nuevos acercamientos ofrecen una imagen más dinámica y cambiante del modo en que migrantes y no migrantes organizan su vida familiar a lo largo del tiempo, en relación a las transformaciones del proyecto migratorio y a las limitaciones impuestas por los Estados (Pedone, Echeverri, Gil Araujo, 2014; Echeverri, 2014; de la Torre Ávila 2014).

\subsection{LA MIGRACIÓN DOMINICANA Y BRASILEÑA HACIA ESTADOS UNIDOS Y LA EUROPA MEDITERRÁNEA: BREVE REPASO POR EL ESTADO DE LA INVESTIGACIÓN}

Las migraciones dominicanas y brasileñas han sido analizadas desde distintas perspectivas y con énfasis en diferentes aspectos. Gran parte de la producción científica en torno a la migración dominicana se ha enfocado en las estrategias de 
reproducción social de los hogares, mientras que en los estudios sobre migración brasileña las relaciones familiares son un campo de estudio apenas abordado.

El corpus de estudios sobre la migración dominicana en Estados Unidos es amplio y diverso ${ }^{6}$. Las investigaciones posteriores a los años ochenta, además de prestar atención a factores económicos y políticos en ambos contextos, evidenciaron la importancia de los vínculos familiares y las cadenas migratorias como factor explicativo de la continuidad de las migraciones entre la República Dominicana y Estados Unidos (Pessar 1987; Portes y Guarnizo 1991). El estudio pionero de Grasmuch y Pessar (1991) advertía sobre la necesidad de cuestionar la visión armoniosa de los núcleos familiares y prestar atención a las jerarquías de género y generación que los atraviesan. Centrándonos en la perspectiva transnacional, la obra de Basch, Glick Schiller y SzantonBlanc (1994) toma el transnacionalismo dominicano a Estados Unidos como uno de sus casos de estudio. Otros antecedentes de importancia son los trabajos de Guarnizo (1997) y la obra de Levitt (2001). En el caso de España, desde la antropología feminista, Gregorio Gil (1998) analizó los cambios que la migración internacional provoca en las dinámicas de género tanto en origen como en destino a partir de la emigración de mujeres dominicanas. Yolanda Herranz (1996) abordó las diferentes formas de incorporación laboral de la inmigración dominicana en Madrid. Los estudios de Nina Sorensen (1999), pioneros en el análisis de la migración dominicana a España, prestaron atención al transnacionalismo familiar.

En contraste con la producción de los años noventa, es llamativa la escasez de nuevos estudios sobre la migración dominicana desde principios del siglo XXI. Entre las pocas excepciones, destacamos los trabajos de Oso y Villares (2005) sobre el empresariado étnico, la investigación de Sorensen y Guarnizo (2007) sobre el transnacionalismo familiar de la población colombiana y dominicana en Europa, el informe inédito de García O’Meany (2009) sobre las experiencias de socialización de hijos e hijas de familias de origen dominicano en Cataluña y el artículo de Pedone, Agrela y Gil Araujo (2012) sobre la incidencia de las políticas públicas y las relaciones de género en las estrategias de reunificación familiar de las migrantes procedentes de Bolivia y República Dominicana. Se sabe poco sobre las formas que adquiere el transnacionalismo familiar entre República Dominicana y España, en el contexto de una migración consolidada, que se inició hace tres décadas.

A fines de la década de 1990 aparece el primer corpus de estudios sobre la migración brasileña en Estados Unidos. Uno de los casos más estudiados es la migración de la ciudad Gobernador Valadares (Minas Gerais) hacia Boston, que se inició en los años ochenta. Las investigaciones se han centrado en mayor medida en la inserción laboral, remesas y redes migratorias (Sales 1991). En los últimos años se han publicado estudios sobre las migraciones familiares, las modalidades de reagrupación, la inserción de los/as hijos/as de origen brasileño (Sales y Loureiro, 2004), las formas de organización familiar y las redes migratorias femeninas (Assis de Oliveira 2004).

\footnotetext{
${ }^{6}$ En 1992 se fundó en Nueva York, el Dominican Studies Institute, en el City College, el primer organismo universitario dedicado al estudio de la población de origen dominicano asentada en Estados Unidos (Sorensen, 2005).
} 
En el contexto europeo, históricamente el principal destino de la migración brasileña ha sido Portugal, debido a los lazos coloniales y neocoloniales establecidos entre los dos países. Las investigaciones se centran principalmente en los efectos que los acuerdos bilaterales tienen en estos desplazamientos y en la inserción laboral (Padilla, 2007; Massanet y Padilla, 2010; Pinho, 2010). Dentro de esta temática general, existe un corpus de estudios que enfatizan en los marcadores de género y de la racialización/etnicización, comunes a las experiencias de todas las mujeres migrantes brasileñas (Padilla et. al, en prensa). También han sido objeto de investigación los matrimonios binacionales entre hombres portugueses y mujeres brasileñas (Bógus, 2007) y el tráfico de personas hacia Portugal (Peixoto et. al., 2005; Peixoto, 2007). Otras investigaciones centran el análisis en la inserción de las mujeres brasileñas en trabajo doméstico y de cuidados, la estética, el sector de ventas y la atención al público (Padilla, 2007; Pereira, 2010), tareas que algunos autores identifican como el mercado de la alegría o mercado de la simpatía (Machado, 2004).

Una perspectiva similar siguen los estudios llevados a cabo en el contexto español, mayoritariamente enfocados en la inserción laboral (Cavalcanti 2003, 2010; Carvalho, 2010; Solé, Cavalcanti y Parella, 2011). Resaltamos que los estudios de la migración brasileña con enfoque de género han privilegiado el análisis de los matrimonios binacionales, el turismo y el trabajo sexual, desde la interseccionalidad entre sexo, etnia y clase, así como en las representaciones sociales de la mujer brasileña a partir de las concepciones sobre afectividad, racialización, sexualización y occidentalización (Piscitelli, 2007; Roca et.al, 2010; De Souza Badet, 2010). También se han abordado los arquetipos masculinos en el análisis de la capoeira en los contextos de destino (Guizardi, 2011).

En los últimos años, a partir de la crisis socioeconómica global, los estudios analizan las estrategias de retorno de la población brasileña desde Europa y Estados Unidos en general y desde una perspectiva de género en particular (Alburquerque Ferreira et al., 2014; Botega y Ruano, 2015). Por el momento, las formas de organización familiar y los reacomodamientos de las relaciones de género y generaciones en la migración brasileña continúan sin ser abordadas en profundidad.

\section{FAMILIAS TRANSNACIONALES DOMINICANAS Y BRASILEÑAS: PROYECTOS MIGRATORIOS, REAGRUPACIÓN FAMILIAR Y ORGANIZACIÓN DE LOS CUIDADOS}

Analizar las formas de organización de las familias dominicanas y brasileñas en un contexto migratorio nos ha permitido identificar algunas similitudes. En ambos casos las mujeres han sido las iniciadoras de la mayoría de los proyectos migratorios familiares y las principales responsables de la gestión de la vida cotidiana en distintos espacios sociales simultáneamente. No obstante, al estudiar estas estrategias teniendo en cuenta el género, la generación, los momentos de llegada, los estatus jurídicos, la procedencia regional, las adscripciones de clase social en origen, el tipo de proyecto migratorio (familiar o individual) y la incidencia de las políticas migratorias observamos un paisaje heterogéneo, tanto en los lugares de origen como en los de destino. 
En esta oportunidad centramos la atención en tres aspectos del proceso migratorio: las características del proyecto migratorio; las formas de organización del cuidado familiar y las estrategias de reagrupación, sin dejar de reconocer que existen otros factores que intervienen en las formas de organización familiar, como por ejemplo la inserción laboral. Tomamos en cuenta la dimensión temporal con el objetivo de reconstruir el modo en que las configuraciones familiares y los patrones migratorios han ido cambiando a lo largo del tiempo. Al hacer hincapié en el cuidado familiar, más allá de visibilizar la relación de la madre migrante que envía remesas y organiza el cuidado en torno a las abuelas, nos interesa mostrar que en estos proyectos se han construido otros vínculos familiares -abuelas y nietos, el rol de los hermanos mayores, los lazos de parentesco no sanguíneos- a los cuales la literatura especializada apenas ha prestado atención. Además, centrar la atención en estas relaciones nos permite dar cuenta del carácter dinámico de las formas de organización familiar transnacional, que en algunos casos, se extienden mucho más tiempo del previsto en el inicio del proyecto migratorio.

\subsection{PROYECTOS MIGRATORIOS: MUJERES COMO PRIMER ESLABÓN DE LA CADENA MIGRATORIA}

En relación a los proyectos migratorios encontramos algunas similitudes entre las mujeres dominicanas y brasileñas entrevistadas, pertenecientes a una clase social baja o media baja que optan por la migración internacional como una estrategia de supervivencia o de mejora en la calidad de vida. Estos proyectos se caracterizan por tener a las mujeres como primer eslabón de la cadena migratoria, mujeres que encabezan familias monoparentales y que posteriormente se convierten en jefas de grupos domésticos transnacionales.

La migración dominicana hacia Madrid y Barcelona fue el primer flujo feminizado que llegó a España a mediados de 1980. Dos de los factores que impulsaron este proceso fueron a) el inicio de una demanda de mano de obra femenina para los servicios de proximidad en destino y b) la existencia en origen de grupos domésticos matrifocales, donde los varones están ausentes o tienen varios hogares (Gallardo 1995, Gregorio 1998).

El espesor histórico de este flujo ha permitido a las mujeres dominicanas, sobre todo a las pioneras, desplegar una diversidad de estrategias migratorias a lo largo del tiempo: obtener la ciudadanía española, organizar migraciones circulares en torno al cuidado de mayores e hijos/as y ensayar diferentes modalidades de reagrupación de menores y maridos, como veremos más adelante.

En nuestra investigación sobre la migración brasileña identificamos una diversidad de estrategias. El trabajo de campo exploratorio en Brasil (Pedone y Alonso, 2010) nos permitió observar una notable heterogeneidad en los proyectos migratorios, marcados principalmente por el género, la edad, la pertenencia de clase y la procedencia regional. Tanto los/as entrevistados/as vinculados/as a la migración, como informantes claves e investigadores/as expertos en migración en Brasil, coinciden en señalar que las familias o mujeres de clase baja o media baja que se suman a la 
migración internacional como una estrategia de supervivencia, proceden de los estados de São Paulo, Minas Gerais, Goiás, Bahía, Rondônia, Rio Grande do Sul, Santa Catarina, Pernambuco y Ceará. En estos proyectos migratorios prevalecen dos estrategias diferenciadas, los que contemplan la reagrupación familiar en destino, que privilegia el acceso a la educación de los y las hijas (Pires, 2007), y aquellos en los que se decide no reagrupar, lo que conlleva una consolidación de los procesos de transnacionalismo familiar. Este segundo tipo de proyecto está vinculado con una migración de carácter claramente económico, que suele estar liderada por mujeres con cargas familiares que se convierte en jefas de grupos domésticos transnacionales.

"Por la experiencia que tengo, y porque como soy de Río de Janeiro y pasan muchos pasaportes por mis manos, en todo este período son poquísimos los pasaportes que pasan por mis manos de personas que vienen de Río de Janeiro. La gran masa de migración brasileña es de Rondônia, Goiás, Minas Gerais, Bahía y Espíritu Santo. Mientras que las personas que vienen de San Pablo son aquellos que tienen un vínculo con España, nietos de españoles, descendientes de españoles que migran de San Pablo. (Abogada brasileña, gestora de trámites de migración residente en Barcelona)".

En concordancia con el testimonio precedente, nuestro trabajo de campo nos reveló que la migración desde el estado de Río de Janeiro hacia España está compuesta por varones y mujeres, jóvenes, cualificados/as, con un proyecto individual de perfeccionamiento profesional en destino que les permita retornar y situarse en una mejor posición socioeconómica en los lugares de origen. Para llevar a cabo este proyecto migratorio se despliegan principalmente dos estrategias: a) tramitar la nacionalidad española o de algún otro país europeo (Italia, por ejemplo) por descendencia o por casamiento y b) recibir remesas procedentes de sus progenitores que permanecen en origen. Generalmente se trata de familias de clase media, media alta, con padres profesionales, que ven en estos proyectos una forma de inversión y capitalización socioeconómica y cultural. Algunos de nuestros testimonios dan cuenta de otra estrategia que organiza el proyecto migratorio de una parte importante de las mujeres brasileñas: el casamiento con varones europeos, en este caso, españoles. Estos hallazgos en Barcelona se corroboran con otros estudios llevados a cabo en Madrid (Guizardi, 2013), Portugal (Padilla, 2007, 2009), Italia (Piscitelli, 2008) y Alemania (Lidola, en prensa).

\section{2. "SIEMPRE HAY ALGUIEN QUE PIENSA, A MÍ NO ME HAN LLEVADO...”: PROCESOS DE REAGRUPACIÓN FAMILIAR}

"Las dominicanas prefieren reagrupar a sus hijos antes que a sus maridos, prefieren tener a sus maridos en Santo Domingo. No es como en el caso ecuatoriano que las mujeres reagrupan a la mayor parte de su familia y sobre todo a sus maridos. Las dominicanas casi no reagrupan a sus maridos, es curioso a veces no te dicen que están casadas y para reagrupar a sus hijos hay que traer esos papeles. Las brasileñas generalmente no suelen reagrupar, más aún aquellas que están en el trabajo sexual y sus familiares en Brasil no lo saben. Además, los 
varones no están presentes son redes familiares femeninas, suelen dejar sus hijos con sus madres, ellas vienen solas, de Rondônia, Goiás, la mayoría no trae a sus hijos. Yo incluso les aconsejo que no traigan a sus hijos, porque no tendrán tiempo de estar con ellas, y se irán con malas compañías, mejor tener a tu hijo allá que le des una buena educación en Brasil pero no traerlo aquí para estar... muchos han tenido que retornar, no alcanzan a darle la atención adecuada. Algunas brasileñas, tienen la suerte de encontrar a un hombre, se casan y dejan el trabajo, yo conozco a muchas que están en la casa con sus maridos”. (Abogada brasileña, gestora de trámites de migración residente en Barcelona)

Debido a los diferentes momentos de llegada de la migración dominicana y brasileña hemos podido distinguir diferentes estrategias de reagrupación familiar que generan nuevas formas de organización familiar y de ejercer el cuidado. En el caso de la migración dominicana, la entrada en vigor del visado a partir de 1992, obstaculizó el ingreso a España por vías informales. A partir de esa nueva realidad normativa una de las estrategias más frecuentes encontradas en nuestro trabajo de campo es la reagrupación familiar mediante la utilización de las cuotas del mercado de trabajo o contingentes. Esta vía alternativa, también utilizada por la migración ecuatoriana y colombiana, se convirtió en la herramienta para traer a parientes no incluidos en la reglamentación de la reagrupación familiar, como hermanas, hijos mayores de 18 años, tías y demás miembros de la familia extensa.

Además, la migración dominicana lleva casi dos décadas de procesos de reagrupación familiar que han visibilizado y en ocasiones estigmatizado a los hijos e hijas.

"Mira todo era muy diferente que ahora, cuando llegamos éramos todas mujeres, vivíamos en pisos que alquilábamos entre todas. Todas trabajábamos en el servicio doméstico interno, y los fines de semana íbamos para el piso que compartíamos.Uno no venía con contrato sino que entraba como turista, en el '92 entró en vigor la visa para los dominicanos. Mira, cuando yo vine ya habían dominicanas que ya tenían más de 10 años por aquí. A partir del '94, ya las cosas fueron cambiando porque ya fueron trayéndose a las familias, entonces estos pisos de mujeres solas se iban desarmando porque había que buscar vivienda para la familia. Se traían primero a los hombres. Puedo darte fe que a partir de 1995 vienen llegando los chicos. Llegan de todo, adolescentes, llegan pequeños, los pequeños van bien en la escuela, los adolescentes no todos”. (Mujer, 45 años, procedente de Los Ríos, Entrevista en Barcelona)

La información que circula entre las generaciones más jóvenes proviene de largos períodos de separación y negociaciones familiares e interviene en las nuevas miradas y relecturas que ellos elaboran sobre los proyectos migratorios familiares.

"Soy de la República Dominicana y tengo 19 años. La primera fue mi madre, vino hace mucho en el '90. Yo vine por primera vez cuando tenía trece años, para estar con ella. Allá estaba con mi abuela, mi hermana se quiso quedar con mi abuela. Luego me volví porque quería estudiar la Universidad allá, luego volví porque ya tenía papeles, luego regresé a Dominicana, ahora volví a pasear y me vuelvo 
nuevamente. Ahora ya mismo no sé dónde quiero estar. Estudio Administración de Empresas y quiero terminar la carrera allí y luego venir para acá para trabajar”. (Mujer, 19 años, procedente de Santo Domingo, Entrevista en Barcelona)

Desde el comienzo de la actual crisis económica que afecta de manera particular a la Europa Mediterránea, en España los procesos de reagrupación familiar se han reducido de manera drástica (Pedone, Echeverri, Gil Araujo, 2014). Por un lado, debido a la dificultad de las migrantes para cumplir con los requerimientos de permiso de residencia, contrato de trabajo, ingresos y vivienda. Pero también por la ralentización del trámite administrativo y el incremento de las denegaciones a las solicitudes de reagrupación, así como a las renovaciones de permisos de residencia y trabajo y a las regularizaciones por arraigo. En los últimos años, debido a que la regulación de la reagrupación se ha convertido en un instrumento de control de los flujos. Nuestro trabajo de campo tanto en los lugares de emigración como de inmigración, nos ha permitido constatar la existencia de prácticas formales e informales que obstaculizan el derecho a la vida en familia, cuya aplicación depende de los funcionarios de turno y de los casos específicos que presentan la documentación:

“Antes tú podrías traerte a una persona, no exigían tantos documentos, y podías traer a una persona incluso con la documentación de otra. Me dijeron a mí, tú con 33 años y que tengas una niña de 17 años... no me creían, pensaban que era mi hermana pequeña. Entonces me exigen una prueba de ADN. Yo tuve que hacerme una prueba de ADN aquí en un hospital especial que te mandan ellos y cuesta unos 200 euros. Entonces esa prueba de ADN a la embajada española directamente. Allí ellos mandan a las niñas a una clínica también especial para eso. Todo eso lo pago yo, todo eso sale de mi trabajo. Una prueba de ADN a ella y a las otras dos menores, que me costó casi 1000 euros. Luego que vieron que la prueba de ADN era positiva, que estaba todo bien, entonces ellos decían que... como ella nació en el 94 pero yo la declaré en el 95. Fue declarada tardía. Entonces al tener un año de retraso por declaración porque cuando yo la tuve a ella, yo era menor de edad, no tenía DNI todavía. Al ser así se retrasó un año su libro de familia. Entonces a la más grande tuvieron que hacerle pruebas de huesos. A ella y a la menor, porque a la menor me pasó igual, pero la menor fue por descuido mío, no estaba con su padre, ya nos habíamos separado, y el libro de familia se retrasó”. (Mujer, 34 años, procedente de Santo Domingo, Entrevista en Barcelona)

En el caso de la migración brasileña la preeminencia de mujeres jóvenes jefas de hogar, y con alto porcentaje de irregularidad hace que los procesos de reagrupación sean escasos. Si bien el no requerimiento de visado posibilita viajar a España con mayor capacidad de movilidad, a su vez, pasado los tres meses como turista, la mayoría de estas migrantes caen en la irregularidad jurídica, con muy limitadas posibilidades de obtener permiso de trabajo y residencia ${ }^{7}$. En un flujo migratorio tan

${ }^{7}$ Actualmente la única vía de regularización es por arraigo social, a la que se puede acceder a partir de los tres años de residencia continuada en España, que debe ser demostrada. 
reciente, la inexistencia de procesos de regularización desde el año 2005 ha influido en la consolidación del transnacionalismo familiar, ya que la reagrupación de hijos e hijas en destino no es una estrategia prioritaria para este colectivo de mujeres migrantes. En este sentido, otro factor de importancia es la inserción de alguna de estas mujeres en el trabajo sexual (Juliano, 2010).

Los testimonios que resumimos aquí ejemplifican de qué manera la exigencia o no de visado, los procesos de regularización, las vinculaciones entre el permiso de trabajo y el permiso de residencia, la regulación de la reagrupación familiar, así como las precarias condiciones laborales, residenciales y el contexto socio-educativo en los lugares de destino, inciden en las modalidades de reagrupación familiar y dan lugar a diversas formas de organización del cuidado, como veremos a continuación.

\subsection{ORGANIZACIÓN DEL CUIDADO: MIGRACIONES CIRCULARES, NUEVAS RESPONSABILIDADES, REDES DE MUJERES}

En la migración dominicana las estrategias de organización familiar han cambiado a lo largo del tiempo. Los primeros arreglos familiares organizaban el cuidado de los hijos entre las madres migrantes y las abuelas que permanecían en origen. Posteriormente, la obtención de la nacionalidad española dio lugar a migraciones circulares que permiten optimizar la inversión de los recursos económicos y programar los estudios de los/as jóvenes. En esta etapa es necesario garantizar el cuidado de los mayores que han envejecido, pero no siempre quienes migraron asumen esta responsabilidad una vez que reagruparon a sus hijos en destino. Una vez más, es cuestión de género. Las abuelas entrevistadas en República Dominicana exponen diferentes situaciones cuando se trata de hijos o hijas migrantes.

Generalmente, las mujeres migrantes siguen enviando remesas a sus madres y organizando el cuidado de las personas mayores a nivel transnacional, como lo hicieron con sus hijos, cuando ellas encabezaron la migración. Así lo describe esta abuela:

"Ay sí. Esas nunca, nunca se olvidaban de mandar ese dinero. Ese dinero cada mes ahí estaba. Y su ropa, cuando encontraban con quien mandárselas, también la mandaban. Esas nunca se descuidaron de sus hijos, su ropa, sus zapatos y no llegaban con las manos vacías. Nunca me faltó, aunque yo trabajaba también aquí, yo lavaba y planchaba en casas de familia. Ahora ellas si siguen mandando. Si necesito una medicina me la mandan de allá, o me mandan el dinero para que yo la compre aquí. No se descuidan de mí, siempre están pendientes de su madre”. (Mujer, 65 años, crió a 7 nietos de sus hijas e hijo migrantes en España, Entrevista en Santo Domingo)

Sin embargo, en nuestras entrevistas, las abuelas de familias con proyectos migratorios iniciados por varones no reciben más remesas una vez que terminaron sus tareas de cuidados. Las justificaciones aluden a cuestiones de género. Las mismas abuelas entienden que es más difícil que sus hijos envíen dinero porque ya hicieron una familia: 
“...esa fue una decisión que él tuvo... migrar y no le está yendo del todo bien. La situación en que él está... no es más o menos como se pensaba, la situación de trabajo no ha sido buena. Yo creo que él no pensará volver porque él está haciendo sus papeles, su vida por allá. Tiene su familia allá. Él hizo vida por ahí. Vino una vez, el año pasado, vino con la beba, su esposa y su suegra. Ya Alex, es mi hijo verdad, pero ya él hizo vida por allá”. (Mujer, 54 años, su marido migró a Estados Unidos y su hijo a España, Entrevista en Santo Domingo)

Llama la atención comprobar que las mujeres mayores entrevistadas no reclaman cuidado. Frente a la falta de estrategias por parte de sus hijas e hijos para reagruparlas o garantizar su cuidado en los lugares de origen, argumentan que sus hijas y, sobre todo, sus hijos ya tienen demasiadas responsabilidades familiares en los lugares de emigración como para encargarse de ellas. Con el agravante que la mayoría de las abuelas entrevistadas en República Dominicana viven en una situación de profunda precariedad económica y residencial, sin posibilidades de cobrar pensiones o jubilaciones en su vejez.

“¿Nunca pensaron sus hijas en llevarla a usted a España?

$\mathrm{Si}$, ellas me han depositado dos veces. Pero me lo deniegan. Unas de mis hijas me depositó cuando reagrupó a la niña que yo tenía desde los dos años. Que era para que yo me vaya junto con ella. Me lo negaron. Después la otra hija también me depositó, al lado de su esposo que estaba aquí. Le salió a su esposo, y a mí no me salió. No me dijeron por qué. Yo pensé que era porque era dizque de 65 en adelante y yo no tenía mis 65 . Pues, yo pensé que era por esta enfermedad, que ni me lo explicaban. Ahora ya no quiero ir, porque ya sentada allá y sentada aquí... ellas si trabajan, no me van a atender. Entonces para estar de más por allá, es mejor quedarme aquí en mi país. Cualquiera que me conoce viene y me ayuda, o si no me llevan a sus casas y ahí estoy cuidada. Si yo iba era para estar al lado de mis nietos, para cuidarlos. Pero ya mis nietos están grandes, ya no hay que cuidarlos. Y tampoco ellos me pueden cuidar a mí, ellos están estudiando, trabajan, ya no me pueden cuidar”. (Mujer, 65 años, sus hijas migrantes en Barcelona, Entrevista en Santo Domingo)

En otros casos, la permanencia dilatada de mujeres dominicanas en el servicio doméstico ha extendido los tiempos de separación por más de una década. En este contexto migratorio hemos detectado el traspaso generacional de responsabilidades desde adultos, como abuelas o tías cuidadoras, hacia jóvenes mayores, que a cierta edad dejan de estar bajo la tutela de otros familiares y comienzan a ser responsables del proyecto migratorio familiar en origen, específicamente en lo que respecta al manejo de la casa y de la inversión de las remesas que envían sus madres.

"Primero, nos quedamos con la prima que vivía con nosotros, casi 10 años viviendo juntos.... Cuando ella volvió, por primera vez después de tres años fue ahí cuando nos cuenta los motivos que tuvo, los planes, las frustraciones que nunca tuvo allá que tuvo aquí... Entonces después de que vino, decidimos ya mi hermano y yo vivir solos. Entonces, mi madre quería buscar ayuda porque en esos 
tiempos yo apenas llegaba a los 18 años y dejar un muchacho de 18 y un niño de $16,15 \ldots$ no es recomendable. Así que nuestra hermana se muda con nosotros, pero a los dos años ella se consigue un novio, se casa, y ya mi hermano y yo somos prácticamente adultos. Ya mi madre toma la decisión que nosotros podemos solos, que podemos administrar la casa por nosotros mismos y así nos hemos quedado hasta el día de hoy”. (Varón, 21 años, hijo de madre migrante en España, Entrevista en Santo Domingo).

En las migraciones familiares brasileñas, las mujeres jefas de hogar migran con la idea de un retorno a medio y largo plazo y privilegian una organización del cuidado transnacional. El envío de remesas a los familiares dependientes tiene un lugar central en el proyecto migratorio. Si bien, la prioridad son los hijos y las abuelas, también los lazos de la familia extensa tienen un peso importante. Las tías ocupan un rol central en el mantenimiento económico de sus sobrinos y llegan a convertirse en las organizadoras de los hogares.

“Ayudé mucho a mis sobrinos... uno que quedó desempleado y tenía que seguir pagando la facultad, hasta que no se estabilizó le pagué la facultad desde aquí, si hace falta algo hablan conmigo directamente, saben que conmigo no dan nunca vueltas. Ahora no mando dinero. Mi madre siempre mandaba, hasta 2008 desde que llegué siempre mandaba cosas aquí en la época de rebajas de verano pues yo compraba muchísimas cosas que cuesta un euro, mandaba cajas de cosas, para mis sobrinos...” (Mujer, 32 años, precedente de Sao Paulo, seis años en A Coruña)

“A mi padre le mando todos los meses, tengo un valor que mando todos los meses para que pague su plan de salud...allí si quieres un médico bueno entonces mi padre tiene un seguro privado y entonces le mando para que le haga falta”. (Mujer, 33 años, procedente de Rio Grande do Sul, Entrevista en A Coruña).

En el caso de la migración brasileña de jóvenes cualificados/as con proyectos individuales de perfeccionamiento profesional, las remesas circulan en sentido inverso. Son los padres, desde origen, los que envían dinero y están atentos a la satisfacción de necesidades y deseos de sus hijos, que apuntan a reforzar los objetivos de inversión en capital cultural.

"Quería profundizar en la lengua española, estando en Brasil no podía no era suficiente las horas de español que tenía en la Universidad. Mi padre me tenía que enviar una parte para poder pagar el alquiler y como no me llegaba, trabajaba como camarera unas 10 horas, iba a la universidad por la mañana y por tarde y organizaba para tener 4 días al final del mes para viajar. Mi tarjeta de crédito es internacional, mi padre me depositaba el dinero allí y yo lo cogía aquí, así cubría el alquiler de la habitación, alimentación y transporte....era menos de lo que normalmente te dicen cuando vas a Europa para vivir. Pero como estudiante y con 350 euros y yo trabajaba para cubrir lo demás, porque mientras trabajaba como camarera ganaba 900 así que me daba para viajar y vivir. El último mes sólo me dediqué a viajar por Europa y en el tiempo que estuve recorrí 14 países”. (Mujer, 23 años, procedente de Río de Janeiro, estuvo un año en A Coruña, Entrevista en Río de Janeiro) 
En síntesis, el análisis de los proyectos migratorios, las estrategias de reagrupación familiar y la organización del cuidado en la migración dominicana y brasileña hacia España nos permitieron mostrar la heterogeneidad que presentan las formas de organización de las familias migrantes. Nuestra finalidad no es caracterizar la totalidad de estrategias de estos colectivos, sino visibilizar su diversidad según el género y generación, la clase social, la procedencia regional y la dimensión temporal.

\section{CONCLUSIONES}

En este artículo hemos indagado los proyectos familiares, las estrategias de reagrupación y la organización del cuidado en el contexto de la migración desde Brasil y República Dominicana hacia España. Nuestro objetivo ha sido mostrar la heterogeneidad y dinámica de las formas de organización familiar, que cambian a lo largo del proyecto migratorio y están moldeadas por las políticas migratorias, los estatutos jurídicos de los migrantes, pero también por el ciclo vital. Tener en cuenta el género, la generación, la clase social, y la procedencia regional nos permitió dar cuenta de la diversidad, no sólo entre flujos migratorios de distinto origen nacional, sino relevar las diferencias y desigualdades que atraviesan a las poblaciones migrantes, tanto en los contextos de origen como de destino. Estos hallazgos cuestionan el lugar preponderante que se le suele otorgar al origen nacional en el estudio de los procesos migratorios internacionales.

En cuanto a la organización del cuidado, nuestro trabajo de campo etnográfico transnacional y de corte longitudinal en República Dominicana nos posibilitó trascender el estudio del vínculo materno-filial y visibilizar el papel que cumplen otros integrantes de los grupos domésticos transnacionales: las tías y los/as hermanos/as mayores como administradores de las remesas y las abuelas que con el paso del tiempo se convierten en demandantes de cuidado.

Al prestar atención a la pertenencia de clase social verificamos la existencia de proyectos migratorios individuales, de jóvenes brasileños cualificados que migran a España para mejorar su formación profesional. En estos casos la maternidad y paternidad transnacional se ejercen con hijos adultos, en sentido origen-destino, inverso al que ha sido investigado tradicionalmente.

Los/as migrantes que acceden a la nacionalidad española tienen menos constreñimientos para migrar a otros destinos, retornar o desplazarse aprovechando las ventajas del espacio social transnacional. El espesor histórico del flujo dominicano nos permite distinguir migraciones circulares entre España y República Dominicana, que en el caso brasileño aún son minoritarias, salvo si hablamos de los migrantes de clase media acomodada con doble nacionalidad. Esta circularidad en las migraciones dominicanas permite reorganizar el cuidado de un modo más flexible, alternando estancias entre origen y destino. Estas dinámicas se asemejan a las identificadas en los estudios de la migración dominicana a Estados Unidos.

Por último, en las investigaciones que hemos realizado en la última década diferenciamos y caracterizamos las modalidades de reagrupación familiar: 
reagrupación escalonada, consolidación del transnacionalismo, desagrupación familiar en el contexto del retorno, circularidad entre origen y destino que en algunos casos puede incluir un tercer país, como Estados Unidos y recientemente, otros países europeos.

\section{REFERENCIAS BIBLIOGRÁFICAS}

ALBURQUERQUE FERREIRA, SUELA.; GOMES TEIXEIRA, TERESA.; RAU DE ALMEIDA CALLAU, MANUELA y RICARDO, JUAN PABLO (2014): Inmigrantes brasileñas emprendedoras en Portugal y en Brasil. Impactos de la crisis y del retorno. En Zabala de Cosío, M.E. y Rozée Gómez, V. coords., El género en movimiento. Familias y migraciones, 221-238.México D.F.: El Colegio de México.

ARIZA, MARINA (2000) Ya no soy la que dejé atrás... Mujeres migrantes en República Dominicana. México: Instituto de Investigaciones Sociales/Editorial Plaza

ASSIS DE OLIVEIRA, GLÀUSSIA (2004):De Criciúma para o mundo: os novosfluxos da populaçao brasilera e os rearranjos familiares e de genero, Tesis Doctoral en Ciencias Sociales, Instituto de Filosofía y Ciencias Humanas, UNICAMP, Campinas, San Pablo (inédito)

BAENINGER, ROSANA (2002): La migración internacional de los brasileños: características y tendencias. Santiago de Chile: Centro Latinoamericano y Caribeño de Demografía (CELADE) - División de Población.

BALDASSAR, LORETTA; BALDOCK, CORA y WILDING, RAELENE (2007): Family Caring Across Borders. Migration, Ageing and Transnacional Caregiving.Palgrve/McMillan

BASCH, LINDA., GLICK SCHILLER, NINNA.; SZANTON BLANC, CRISTINA (1994): Nations Bounded. Trasnational Projects, Poscolonial Predicaments and Deterritorialized Nation-States.EE.UU.: Gordon and Breach Science Publishers.

BASTIA, TANIA (2009): Women's migration and the crisis of care. Grandmother caring for grandchildren in Urban Bolivia, Gender and Development; vol 17, $\mathrm{n}^{\circ}$ 3, 389-401.

BOGUS, LÚCIA (2007): EsperançaAlém-Mar: Portugal no ArquipélagoMigratório Brasileiro. En Malheiros, J. ed. Imigração Brasileira em Portugal, 39-58. Lisboa: ACIDI, Presidência dos Ministros.

BOTEGA, TUÍLA y RUANO, ELIZABETH (2015): A dimensão do retorno nas narrativas de migrantes paraguaias e brasileiras. En Nogales Vasconcelos, A.M. y Botega, T Orgs., Política migratória e o paradoxo da globalização, 209-234. Porto Alegre: EDIPUCRS-CSEM.

BRYCESON, DEBORAH y LO VUORELA, ULLA eds. (2002): The Transnational Family: New European Frontiers and Global Networks. Oxford: Berg Publishers. 
CARVALHO, FLÀVIO (2010): Crise e Trabalho Imigrantes na Espanha, em perspectiva brasilera. Colectivo Brasil Catalunya. Rede de Brasileiras e Brasileiros na Catalunha. Barcelona. (inédito)

CAVALCANTI, LEONARDO (2003): Los inmigrantes brasileños en Barcelona: un estudio antropológico sobre sus estrategias y su vida cotidiana. Tesis doctoral: Salamanca: Departamento de Sociología, Universidad de Salamanca.

CAVALCANTI, LEONARDO (2010): La migración brasileña en la estructura socioeconómica de España (resumen ejecutivo). 1er. Seminário de Estudos Sobre Imigração Brasileira na Europa. Barcelona, November 2010

DE LA TORRE AVILA, LEONARDO (2014): Más notas sobre el retorno cíclico boliviano. Control y libertad en los proyectos de movilidad entre España y Bolivia. En Sole, C.; Parella, S. y Petroff, A. coords. Las migraciones bolivianas en la encrucijada interdisciplinar: evolución, cambios y tendencias, Focuson International Migration n ${ }^{\circ}$ 1, 125-151. Bellaterra: Universidad Autónoma de Barcelona.

DE SOUZA BADET, MARÍA (2010): El protagonismo de las brasileñas en los medios de comunicación de España". En XV Seminario Académico APEC. América Latina: Diálogos posibles. Barcelona, Junio.

ECHEVERRI, MARÍA MARGARITA (2014): A los dos lados del Atlántico. Reconfiguraciones de los proyectos migratorios y la vida familiar transnacional de la población colombiana en España, Papeles del CEIC, vol. 2014/2, CEIC, Universidad del País Vasco, http://dx.doi.org/10.1387/pceic.12988

GALLARDO, GINA (1995): Buscando la vida: dominicanas en el servicio doméstico en Madrid. Santo Domingo: CIPAF-IEPALA.

GARCÍA O’MEANY, MARGARITA (2009): Experiències de socialitzación de fills i filles de famílies de República Dominicana a Catalunya. Informe de Investigación. Barcelona: Fundació Jaume Bofill. (inédito).

GIL ARAUJO, SANDRA (2009): Civic Stratification, Gender, and Family Migration Policies: An exploratory Investigation of Migrants involved in Family Migration in Spain, NODE interview analysis, Viena: BMWF/ICMPD.

GIL ARAUJO, SANDRA y PEDONE, CLAUDIA (2014): Introducción. Familias migrantes y Estados: vínculos entre Europa y América Latina. Papeles del CEIC, vol. 2014/2, CEIC, Universidad del País Vasco, http://dx.doi.org/10.1387/pceic.13020

GONZÁLVEZ, HERMINIA (2010): Migración colombiana, género y parentesco: La organización social de los cuidados, Tesis doctoral, Granada: Universidad de Granada,.

GRASMUCK SHERRY and PESSAR PATRICIA (1991): Between two Islands. Dominican International Migration. Berkeley: University of Carlifornia Press.

GREGORIO GIL, CARMEN (1998): Migración femenina. Su impacto en las relaciones de género. Madrid: Narcea. 
GUARNIZO, LUIS (1997): Los domincanyorks: the making of a binational society. En Romero, M.; Hondagneu Sotelo, P. y Ortiz, V. eds. Challenging Fronteras. Structuring Latina and Latino Lives in the U.S., 161-175. Nueva York: Routledge.

GUIZARDI, MENRA LUBE (2011): 'COMO SI FUERAN HOMBRES': los arquetipos masculinos y la presencia femenina en los grupos de capoeira de Madrid. Revista de Antropología Experimental, n 11, Texto 21, 299- 315.

GUIZARDI, MENARA LUBE (2013): "Estereotipos, identidades, y nichos económicos de las migrantes brasileñas en Madrid”, Revista de Estudios Feministas, Vol.21, $\quad \mathrm{N}^{\mathrm{o}} \quad$ 1, Florianópolis, Jan./Apr.2013. http://dx.doi.org/10.1590/S0104-026X2013000100009

HERRANZ, YOLANDA (1996): Formas de incorporación laboral de la inmigración latinoamericana en Madrid. Importancia del contexto de recepción. Tesis Doctoral. Madrid: Departamento de Sociología y Antropología Social, Facultad de Ciencias Económicas y Empresariales, Universidad Autónoma de Madrid.

HERRANZ, YOLANDA (1998): La inmigración latinoamericana en diferentes contextos de recepción. Migraciones, 3, 31-51.

HERRERA, GIOCONDA (2008): Políticas migratorias y familias transnacionales: migración ecuatoriana en España y Estados Unidos”. En Herrera, G. y Ramírez, J. coords. América Latina migrante. Estado, familia, identidades, 71-86. Quito: FLACSO, Ministerio de Cultura.

HERRERA, GIOCONDA (2013): “Lejos de tus pupilas”. Familias transnacionales, cuidados y desigualdad social en Ecuador. Quito: FLACSO-Ecuador / ONU Mujeres.

JULIANO, DOLORES (2010): Sacando adelante hijos e hijas. Migración y trabajo sexual. En Grupo Interdisciplinario de Investigador@s Migrantes Coord. Familias, niños, niñas y jóvenes migrantes. Rompiendo estereotipos, 47-54. Madrid: GIIMIEPALA-Casa Encendida-Fundación Social Caja Madrid

LEDO GARCÍA, CARMEN (2014): Múltiples arreglos del hogar transnacional en la ciudad de Cochabamba. Revista Cidob d'affers Internacionals, nº 106-107, 105118.

LEVITT, PEGGY (2001): The Transnacional Villagers. Berkeley: University of California.

LIDOLA, MARÍA: Migración, trabajo y emprendimientos femeninos: empresarias brasileñas y el Brazilian Waxing en Berlín. En Magliano, M. J. y Mallimaci, A. I. comps. Las mujeres latinoamericanas y sus migraciones. Córdoba, Argentina: Editorial Universitaria Villa (EDUVIM) (en prensa).

MACHADO, IGOR (2004): Afetividade e poder entre os imigrantes brasileiros no Porto, Cadernos Pagu, n ${ }^{\circ}$ 23, 257-278.

MARGOLIS, MAXINE (1994): Little Brazil. An ethnography of Brazilian immigrants in New York City. New Jersey: Princeton University Press. 
MASANET, ERIKA Y BAENINGER, ROSANA (2010): Os impactos laborais da criseeconômica sobre a população brasileira na Espanha. 1er. Seminário de Estudos Sobre Imigração Brasileira na Europa. Barcelona, November 2010.

MASANET, ERIKA Y PADILLA, BEATRIZ (2010): La inmigración brasileña en Portugal y España. ¿Sistema migratorio ibérico?.Oberts. Revista de Ciencias Sociales, vol. 5, no.1, 49-86.

MICOLTA LEÓN, AMPARO y ESCOBAR SERRANO, MARÍA CÉNIDE (2010): Si las abuelas se disponen a cuidar, madres y padres pueden migrar. Revista Venezolana de Estudios de la Mujer, julio/diciembre 2010, vol. 15, n³5, 91-116.

OSO, LAURA y VILLARES VARELA, MARÍA (2005): Mujeres inmigrantes latinoamericanas y empresariado étnico: dominicanas en Madrid, argentinas y venezolanas en Galicia. Revista Galega de Economía, vol. 14, nº. 1-2, juniodiciembre, 2005. 1-19.

OSO, LAURA y RIBAS, NATALIA (2012): De la sorpresa a la incertidumbre: abriendo etapas en el estudio de la temática sobre género y migración en el contexto español, PAPERS, 97/3, 511-520.

PADILLA, BEATRIZ (2007): Estado del arte de las investigaciones sobre brasileños y brasileñas en Portugal. En Herrera, G y Yépez Del Castillo, I. eds. Nuevas migraciones latinoamericanas a Europa. Balances y Desafíos. Quito: FLACSOECUADOR-OBREAL-UCL-UB.

PADILLA, BEATRIZ, OLIVEIRA FERNANDES, GLEICIANI MARIA y SELISTER GOMES, MARIANA (en prensa): Ser brasileña en Portugal: inmigración, género y colonialidad. En Magliano, M.J. y Mallimaci, A. comps. Las mujeres latinoamericanas y sus migraciones. Córdoba: Editorial Universitaria Villa (EDUVIM).

PEDONE, CLAUDIA (2006): Estrategias migratorias y poder. 'Tú siempre jalas a los tuyos'. Quito: Abya-Yala-PMCD.

PEDONE, CLAUDIA (2010): Cadenas y redes migratorias: propuesta metodológica para el análisis diacrónico-temporal de los procesos migratorios, EMPIRIA. Revista de Metodología de Ciencias Sociales, nº 19, Enero-Junio 2010, 101-132.

PEDONE, CLAUDIA (2011): Familias en movimiento. El abordaje teóricometodológico del transnacionalismo familiar latinoamericano en el debate académico español, Revista Latinoamericana de Estudios de Familia, vol. 3, enero - diciembre 2011, 223 - 244.

PEDONE, CLAUDIA (2014) Rupturas y continuidades de los roles de género en contextos migratorios transnacionales. Relatos sobre sexualidad y salud reproductiva de los hijos e hijas de la inmigración ecuatoriana en Cataluña. Papeles del CEIC, vol. 2014/2, $\mathrm{n}^{\circ}$ 111, CEIC (Centro de Estudios sobre la Identidad Colectiva), Universidad del País Vasco, http://dx.doi.org/10.1387/pceic.12968 
PEDONE, CLAUDIA y ALONSO PARDO, Paula (2010): La migración brasileña a España: una mirada transnacional. Informe de Investigación. CIIMU-Universidade A Coruña (inédito).

PEDONE, CLAUDIA y GIL ARAUJO, SANDRA (2008): Maternidades transnacionales entre América Latina y el Estado español. El impacto de las políticas migratorias en las estrategias de reagrupación familiar. En Solé, C.; Parella, S. y Cavalcanti, L. eds. Nuevos retos del transnacionalismo en el estudio de las migraciones, 149-176. Madrid: Observatorio Permanente para la Inmigración.

PEDONE, CLAUDIA; AGRELA, BELÉN y GIL ARAUJO, SANDRA (2012): Políticas públicas, migración y familia: una mirada desde el género. PAPERS. Revista de Sociología, nº 97/3, 541-568.

PEDONE, CLAUDIA; ECHEVERRI, MARÍA MARGARITA Y GIL ARAUJO, SANDRA (2014): Entre dos orillas. Cambios en las formas de organización de las familias migrantes latinoamericanas en España en tiempos de crisis global. En Zavala Castelo, M. E. y Rosee, V. eds. El género en movimiento. Familias y Migraciones, 109-138. México: Colegio de México.

PEIXOTO, JOAO; SOARES, ANTONIO; COSTA MARÍA PAULA; MURTEIRA; SUSANA y SABINO, CÁNDIDO (2005): O tráfico de migrantes em Portugal: perspectivas sociológicas, jurídicas e políticas. Lisboa: ACIME.

PEIXOTO, JOAO (2007): Tráfico, contrabando e imigração irregular: os novos contornos da imigração brasileira em Portugal, Sociologia, Problemas e Práticas, $\mathrm{n}^{\circ}$ 53, 71-90.

PEREIRA, SÓNIA (2010): Trabalhadores de OrigemAficanaem Portugal. O impacto das novas vagas na imigração. Lisboa: EdiçõesColibri.

PESSAR, PATRICIA (1987): The Dominicans: Women in Household an theGarmet Industry. En Foner, N. ed. New Immigrants in New York, 103-130. Nueva York: Columbia University Press.

PINHO, ANA FILIPA (2010): Dos “Brasis” para os “Portugais”. Transformações da emigração brasileira nos últimos 20 anos. 1er. Seminário de Estudos Sobre Imigração Brasileira na Europa. Barcelona, November.

PIRES, BIANCA (2007): De la integración deseada a la integración vivida: la experiencia de adolescentes brasileños en escuelas de Barcelona. Tesis doctoral. Barcelona: Departamento de Antropología, Universitat de Barcelona (inédita)

PISCITELLI, ADRIANA (2007): Sexo tropical em um país europeu: migraçao de brasileiras para a Itália no marco do 'turismo sexual' internacional. Estudos feministas, vol. 15, $\quad \mathrm{n}^{\mathrm{o}} \quad 3 . \quad$ Florianópolis, $\quad$ Sept./Dec. 2007. http://dx.doi.org/10.1590/S0104-026X2007000300014

PISCITELLI, ADRIANA (2008): ¿Actuando la 'brasileñidad'? Tránsitos entre circuitos de turismo sexual y los mercados del sexo y matrimonial europeo.Actas del Latin American Studies Association Annual Meeting. Rio de Janeiro. 
PORTES, ALEJANRO y GUARNIZO, LUIS (1991) Capitalistas del trópico la migración en Estados Unidos y el desarrollo de la pequeña empresa en la República Dominicana. Santo Domingo: FLACSO-Republica Dominicana.

PUYANA, YOLANDA., MOTOA, JULIETH Y VIVIEL ADRIANA (2009): Entre aquí y allá. Las familias colombianas transnacionales. Bogotá: UE-Universidad Nacional de Colombia-Fundación Esperanza.

RIBEIRO, GUSTAVO (1998): Goiânia, California. Vulnerabilidade, ambiguidade e cidadania transnacional. Revista Seria Antropologia. Departamento de Antropologia. Instituto de Ciências Sociais. Universidade de Brasília.

RIVAS, ANA MARÍA Y GONZÁLVEZ, HERMINIA (2009): Familias transnacionales colombianas. Transformaciones y permanencias en las relaciones familiares y de género. Barcelona: Cataratas.

ROCA, JORDI; BODOQUE, YOLANDA.; DJURDJEVIC, MARÍA.; MARTÍNEZ, L. AND SORONELLAS, MONSERRAT (2010): Noviazgos transnacionales, migraciones por amor y formación de parejas mixtas en España. En Soronellas, M. ed. Familias en la migración. Emociones, solidaridades y obligaciones en el espacio transnacional, 127-162. Barcelona: Icaria.

SALES TERESA (1991): Novosfluxosmigratórios da população brasileira. Revista Brasileira Do Estudos da Populaçao, Campinhas. 8 (1/2), 21-32.

SALES, TERESA y LOUREIRO, MÁRCIA (2004): Imigrantes brasileiros adolescentes e de segunda geraçãoem Massachusetts, EUA. R. bras. Est. Pop., Campinas, v. 21, no. 2, Jul-Dec, 217-239.

SOLÉ, CARLOTA; CAVALCANTI, LEONARDO Y PARELLA, SÒNIA (2011): La inmigración brasileña en la estructura económica de España. Madrid: Ministerio de Trabajo e Inmigración.

SØRENSEN, NINNA (1999) "The Dominicanization of New York City and Madrid: Transnational Practices and Identifications Reconsidered.” Lateinamerika. 1999. 122

SØRENSEN, NINNA (2005) "Migración, género y desarrollo: el caso dominicano", Zuñiga, Nieves (coord.) La migración, un camino entre el desarrollo y la cooperación, Centro de Investigación para la Paz, Madrid (163-182).

SØRENSEN, NINNA y GUARNIZO, LUIS (2007): La vida de la familia transnacional a través del Atlántico: la experiencia de la población colombiana b dominicana migrante en Europa, Puntos de Vista 9, Género y Transnacionalismo, 7-28. 